\title{
Short-term efficacy and safety of zoledronate acid or denosumab in Japanese patients with postmenopausal osteoporosis
}

\author{
Yumejiro Nakamura ${ }^{1} \cdot$ Tomohiro Shimizu $^{1}$ (1) $\cdot$ Tsuyoshi Asano $^{1} \cdot$ Shun Shimodan ${ }^{2} \cdot$ Hotaka Ishizu $^{1,2}$. \\ Daisuke Takahashi ${ }^{1} \cdot$ Masahiko Takahata $^{1} \cdot$ Norimasa Iwasaki $^{1}$
}

Received: 21 December 2020 / Accepted: 4 March 2021 / Published online: 5 April 2021

(c) The Japanese Society Bone and Mineral Research 2021

\begin{abstract}
Introduction We aimed to compare the efficacy after switching from either bisphosphonates (BPs) or non-BPs (NBPs) to combination therapies of denosumab (DMAb) or zoledronic acid (Zol) with eldecalcitol (ELD) in bone mineral density (BMD) and bone metabolism and investigate the prognostic and risk factors of side effects of this therapy.

Materials and methods One-hundred forty-eight patients with postmenopausal osteoporosis were recruited; their therapy was switched from BPs or NBPs to Zol or DMAb plus ELD (BP-Zol: 43, NBP-Zol: 32, BP-DMAb: 35, and NBP-DMAb: 38). Longitudinal changes in bone metabolic markers (P1NP and TRACP-5b) and BMD were evaluated.

Results In the BP-Zol group, P1NP did not change after 6 months and increased by $38.9 \%$ after 12 months. TRACP-5b decreased $15.8 \%$ after 6 months, but came back to baseline values 12 months after administration. In the rest of the groups, the bone metabolic markers remained suppressed after 6 and 12 months. Compared with baseline, all groups showed increase in BMD after 6 and 12 months. Bone metabolic markers at baseline were correlated with \%change in lumbar spine BMD from baseline to 12 months. P1NP and 25-hydroxy vitamin D levels at baseline were identified as potential predictors of development of acute-phase reactions.

Conclusions The combination therapy of Zol or DMAb and ELD may increase BMD at 12 months after the first administration in Japanese patients with postmenopausal osteoporosis, regardless of BPs pretreatment. Bone metabolic markers at baseline may be useful predictors for reaction to the therapy and side effects caused by these combination therapies in postmenopausal osteoporosis.
\end{abstract}

Keywords Postmenopausal osteoporosis $\cdot$ Eldecalcitol $\cdot$ Denosumab $\cdot$ Zoledronic acid $\cdot$ Bone metabolic marker

\section{Introduction}

Osteoporosis is a chronic, progressive condition that requires long-term management. An estimated 9 million new osteoporosis-related fractures were reported worldwide in the year 2000 [1]. It is reported that 75 million people in the United States, Europe, and Japan are affected by osteoporosis [2]. Oral bisphosphonates (BPs) are commonly prescribed for

Tomohiro Shimizu

simitom@wg8.so-net.ne.jp

1 Department of Orthopaedic Surgery, Faculty of Medicine and Graduate School of Medicine, Hokkaido University, Kita-15 Nishi-7, Kita-ku, Sapporo, Hokkaido 060-8638, Japan

2 Department of Orthopaedic Surgery, Kushiro City General Hospital, Kushiro, Japan osteoporosis [3]; however, inconvenient dosing regimens and side effects can cause low adherence [4], leading to reduced antifracture efficacy $[5,6]$ and increased health care costs [7]. Therefore, extended dosing intervals could improve adherence and establish drug effects [8, 9]. Additionally, due to the COVID-19 pandemic, which caused an unusual cluster of viral pneumonia cases in China that spread globally $[10,11]$, the demand for long-term anti-osteoporosis therapy may increase.

Two injectable antiresorptive agents, denosumab (DMAb) and zoledronic acid ( $\mathrm{Zol})$, have been increasingly used for the treatment of osteoporosis. DMAb is injected subcutaneously $(60 \mathrm{mg}$ ) every 6 months, and Zol is administered intravenously ( $5 \mathrm{mg}$ ) once every 12 months. DMAb, an anti-bone resorptive drug, is a human monoclonal antibody that targets the osteoclast differentiation factor/receptor activator of the NF-kB ligand (RANKL) [12]. Several studies 
have shown that it causes a greater increase in bone mineral density (BMD) and reduction in bone resorption than BPs [13-15]. Zol is a BP that contains an imidazole ring as a side chain, and is the most potent of all the clinically available BPs [16, 17]. In Japan, DMAb and Zol were approved for the treatment of osteoporosis in 2013 and 2016, respectively. Although both drugs have been confirmed for the treatment of osteoporosis in Japanese patients [18-21], there is a lack of clinical evidence regarding a comparison of the efficacy of DMAb and Zol among Japanese patients.

Despite the demonstrated efficacy, several serious adverse effects of DMAb have been reported, including hypocalcemia $[22,23]$ in $2-20 \%$ of women with postmenopausal osteoporosis [24, 25]. To prevent hypocalcemia, short-term $\mathrm{Ca}$ and vitamin D supplements are usually required. Alternatively, Zol, like other BPs, causes acute-phase reactions (APRs), such as pyrexia and myalgia; mostly resolved within 3 days after its infusion [26]. Various risk and protective factors, including race, age, 25-hydroxy vitamin $\mathrm{D}(25(\mathrm{OH}) \mathrm{D})$ levels, and prior BP use leading to the development of APRs have been identified in previous studies [27]. Although a recent study showed that the use of loxoprofen and prior use of BPs in Japanese patients with primary osteoporosis treated with Zol were protective against APRs [28], there is limited information regarding the association of APRs and use of Zol among Japanese patients.

The primary aim of this study was to compare the efficacy after switching from either non-BPs (NBPs) or BPs to the combination therapies of DMAb or Zol and eldecalcitol (ELD) with respect to change in bone mineral density (BMD) and bone metabolism. ELD has a longer half-life, a lower clearance rate, and increased vitamin D receptormediated effects than alfacalcidol [29] among Japanese patients with postmenopausal osteoporosis in a real-world clinical setting. The secondary aim was to investigate the prognostic and risk factors of side effects for patients who switched to DMAb or Zol and ELD.

\section{Materials and methods}

\section{Study design and subjects}

This retrospective study was conducted in accordance with the ethical standards of the Declaration of Helsinki and approved by the Hokkaido University Hospital Institutional Review Board (\#020-0188). Total 115 patients with postmenopausal osteoporosis and high risk of fractures who were treated using DMAb $(60 \mathrm{mg}$, subcutaneously every 6 months $)$ in combination with daily oral ELD $(0.75 \mu \mathrm{g})$ (Edirol; Chugai Pharmaceutical, Tokyo, Japan) from January 2014 to December 2016 and 91 patients who were treated Zol (5 mg, intravenous drip infusion) in combination with
ELD from January 2017 to December 2018 by our clinical team were included (Fig. 1). High risk of fracture was defined as BMD T score $<-2.5$ of standard deviation (SD) and one or more fragility fractures, lumbar BMD T score $<$ -3.3 of SD [30, 31], two or more fragility fractures [30, 32], or semi-quantitative evaluation [33] of existing grade 3 vertebral fracture [32]. The criteria for switching were the lack of increase in BMD despite these treatments. The length of time from the last dose of the previous treatment to the new treatment was one month. Exclusion criteria of this study were (1) switching from selective estrogen receptor modulator and teriparatide; $(2)<50$ years old; (3) male; (4) severe chronic kidney disease (stage 4 and 5); (5) thyroid disease and (6) abnormal serum levels of albumin-corrected $\mathrm{Ca}$ (less than 8.3 or more than $10.3 \mathrm{mg} / \mathrm{dL}$ ) at baseline. A total of 148 patients were enrolled in this study.

The following baseline clinical information was obtained: blood samples for the levels of albumin-corrected $\mathrm{Ca}$, bone metabolic markers, 25(OH)D level, intact parathyroid hormone (PTH), and liver and renal function. BMD was evaluated using dual-energy X-ray absorptiometry (DXA), and $\mathrm{X}$-rays of whole spine and full length of lower limbs were obtained for the evaluation of previous fragility fractures and risk factors of atypical femoral fractures. The levels of serum $\mathrm{Ca}$, total type I procollagen N-propeptide (P1NP), and tartrate-resistant acid phosphatase $5 \mathrm{~b}$ (TRACP-5b) were monitored every 6 months. Monitoring of serum $\mathrm{Ca}$ and inflammatory reactions was started at 1 or 2 weeks after DMAb and $\mathrm{Zol}$ administration to evaluate changes in Ca level and to assess APRs (Fig. 2). The APRs defined in this study were pyrexia, myalgia or arthralgia, headache, malaise, and others with onset within 3 days after Zol administration, as described in a previous study [30].

\section{BMD assessment}

Areal BMD of the lumbar spine (LS; L2-L4), femoral neck (FN), and total proximal femur (TPF) were assessed at

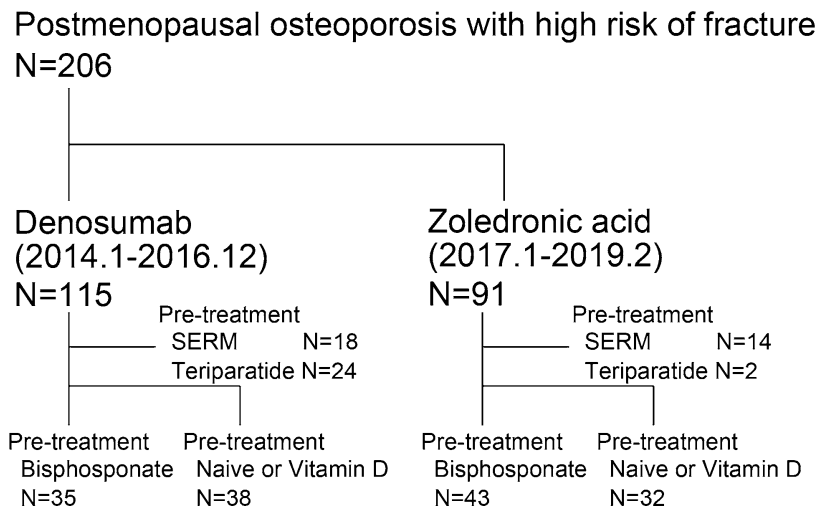

Fig. 1 Study design 
Fig. 2 Clinical protocol

\begin{tabular}{|c|c|}
\hline $\begin{array}{l}\text { Baseline } \\
\text { ( First visit or Switching) }\end{array}$ & $\begin{array}{l}\text { First administration } \\
\text { - Renal and liver function } \\
\text { - Serum } \mathrm{Ca} \text { and } \mathrm{Pi}\end{array}$ \\
\hline - Height and Body weight & \\
\hline - Renal and liver function & One or two weeks after administration \\
\hline - Serum calcium $(\mathrm{Ca})$ and phosphorus $(\mathrm{Pi})$ & $\begin{array}{l}\text { - Blood count, CRP and creatine kinase } \\
\text { - Renal and liver function }\end{array}$ \\
\hline - Total P1NP and TRACP 5b & - Serum $\mathrm{Ca}$ and $\mathrm{Pi}$ \\
\hline - $25 \mathrm{OHD}$ & 1 \\
\hline $\begin{array}{l}\text { - Bone mineral density (BMD) } \\
\text { - X ray } \\
\text { (Whole spine and full length of lower limbs) } \\
\text { - Oral check }\end{array}$ & $\begin{array}{l}\text { Six and twelve months after administration } \\
\text { - Blood test } \\
\text { - Bone metabolic marker } \\
\text { - BMD (Lumbar and Hip joint) } \\
\text { - X ray (whole spine) }\end{array}$ \\
\hline
\end{tabular}

baseline, 6 months, and 12 months after treatment, using DXA Bone Densitometer (Discovery A, Hologic Inc., Massachusetts, USA). Regions of severe scoliosis, previous vertebral fracture, and postoperative sites were excluded from BMD measurements; at least two of the L2-L4 lumbar vertebrae were evaluated for BMD [31]. Subjects were excluded from BMD assessment if the area was fractured or operated on during the study.

\section{Statistical analysis}

Statistical comparisons among the groups were performed using the $\chi^{2}$ test, unpaired $t$-test or a two-way analysis of variance, and Tukey test. Linear regression models adjusted for age, body mass index (BMI), pretreatment with BPs and active vitamin D, 25(OH)D levels, and treatment were established to determine the associations between bone metabolic markers at baseline and \%change of BMD from baseline to 12 months. Multivariate logistic regression analysis, adjusted for age, prior treatment with active vitamin D3, BP use, and acetaminophen, were conducted to determine the factors affecting the development of APRs. All statistical analyses were performed using Statistical Package for the Social Sciences (SPSS Statistics version 23.0) (IBM Corporation, Armonk, NY, USA), with the significance level set at 0.05 .

\section{Results}

\section{Clinical characteristics}

Table 1 shows the baseline characteristics of the patients. The number of patients who switched from BPs to Zol (BPZol), NBPs to Zol (NBP-Zol), BPs to DMAb (BP-DMAb), and NBPs to DMAb (NBP-DMAb) were 43, 32, 35, and 38 , respectively. The mean age of patients in the BP-Zol group was significantly higher than that in the NBP-DMAb group $(P=0.010)$. The mean duration of pretreatment in BP-DMAb group was significantly higher than that in the
BP-Zol group $(P=0.034)$. Total 32 patients in the BP-Zol group, 16 patients in the NBP-Zol group, 23 patients in the $\mathrm{BP}-\mathrm{DMAb}$ group, and 10 patients in the NBP-DMAb group used active vitamin D3 before the administration of ELD. Four patients in the BP-Zol group, three patients in the NBPZol group, three patients in the BP-DMAb group, and two patients in the NBP-DMAb group received oral $\mathrm{Ca}$ before being administered ELD. There were no patients with thyroid or parathyroid abnormalities. The number of patients who experienced fragility fractures was 32, 17, 23, and 21 in the BP-Zol, NBP-Zol, and BP-DMAb groups, respectively. The BP-Zol and BP-DMAb groups exhibited significantly lower P1NP and TRACP-5b levels than the NBP-Zol and NBP-DMAb groups $(P<0.001)$. There were no differences in BMI, Ca levels, 25(OH)D levels, and \%YAM, and BMD at baseline between each group. Notably, during treatment, one patient each in the BP-Zol and NBP-DMAb groups, and two patients in the BP-DMAb group experienced fractures.

\section{Longitudinal changes in Ca, P1NP, and TRACP-5b levels}

The decrease in serum $\mathrm{Ca}$ levels from baseline to 1 or 2 weeks in the NBP groups was significantly higher than that in the BP groups $(P=0.004)$. There were no significant differences in $\mathrm{Ca}$ levels between the DMAb and $\mathrm{Zol}$ groups, and no significant interaction was detected between the treatment and pretreatment groups. Although six patients experienced hypocalcemia $(\mathrm{Ca}$ level $<8.4)$ and one patient experienced hypercalcemia (Ca level $>10.3)$, none experienced serious side effects related to hypercalcemia (short QT syndrome and renal diabetes insipidus) and hypocalcemia (tonic convulsions and tetany). P1NP and TRACP-5b levels at baseline were associated with decreased serum $\mathrm{Ca}$ levels from baseline to 1 or 2 weeks (P1NP: $P=0.003$ and TRACP-5b: $P=0.037$ ) (Fig. 3). In the BP-Zol group, P1NP did not change after 6 months and increased by $38.9 \%$ after 12 months. TRACP-5b decreased $15.8 \%$ after 6 months, but came back to baseline values 12 months after administration. In the rest of the groups, the bone metabolic markers 
Table 1 Clinical characteristics at baseline

\begin{tabular}{|c|c|c|c|c|}
\hline \multirow[t]{2}{*}{ Variable } & \multicolumn{2}{|c|}{ Zoledronic acid } & \multicolumn{2}{|l|}{ Denosumab } \\
\hline & BPs & NBPs & BPs & NBPs \\
\hline Number & 43 & 32 & 35 & 38 \\
\hline Age (years) & $77.6(1.0)$ & $76.6(1.7)$ & $74.4(1.5)$ & $71.8(1.4)^{*}$ \\
\hline BMI $\left(\mathrm{kg} / \mathrm{m}^{2}\right)$ & $22.9(0.5)$ & $21.8(0.6)$ & $22.2(0.6)$ & $22.7(0.5)$ \\
\hline Duration of pretreatment (months) & $28.5(4.0)$ & NA & $43.8(5.9)^{*}$ & NA \\
\hline Prior active vitamin D3 use & 32 patients & 16 patients & 23 patients & 10 patients \\
\hline Prior oral $\mathrm{Ca}$ intake & 4 patients & 3 patients & 3 patients & 2 patients \\
\hline History of fragility fracture & 32 patients & 17 patients & 23 patients & 21 patients \\
\hline $\mathrm{Ca}(\mathrm{mg} / \mathrm{dL})$ & $9.39(0.06)$ & $9.31(0.06)$ & $9.58(0.07)$ & $9.47(0.06)$ \\
\hline eGFR (mL/min/1.73 mm²) & $63.7(2.3)$ & $65.1(2.3)$ & $69.2(3.2)$ & $73.4(2.3)^{*}$ \\
\hline P1NP (ng/mL) & $28.4(3.4)$ & $56.5(7.7)^{\dagger}$ & $21.6(2.0)$ & $56.3(5.4)^{\dagger}$ \\
\hline TRACP-5b (mU/dL) & $307.1(24.5)$ & $411.1(35.0)^{\dagger}$ & $279.5(20.2)$ & $451.0(27.2)^{\dagger}$ \\
\hline $25(\mathrm{OH}) \mathrm{D}(\mathrm{ng} / \mathrm{mL})$ & $14.7(0.8)$ & $14.1(1.3)$ & $17.0(1.2)$ & $17.7(1.0)$ \\
\hline Intact PTH (pg/mL) & $35.0(3.3)$ & $38.6(5.7)$ & $36.8(2.4)$ & $37.1(1.1)$ \\
\hline$\%$ YAM lumbar (\%) & $70.9(1.5)$ & $69.7(2.1)$ & $71.7(2.1)$ & $68.5(2.1)$ \\
\hline \% YAM FN (\%) & $64.2(1.6)$ & $64.3(1.8)$ & $62.2(1.6)$ & $61.8(1.6)$ \\
\hline$\%$ YAM TPF (\%) & $70.5(1.8)$ & $70.2(2.0)$ & $65.9(1.9)$ & $68.0(1.7)$ \\
\hline
\end{tabular}

Mean (standard error of the mean)

$B P s$ bisphosphonates, NBPs non-bisphosphonates, $B M I$ body mass index, $C r$ creatinine, $e G F R$ estimated glomerular filtration rate, $P 1 N P$ total type 1 procollagen- $N$-propeptide, TRACP-5b tartrate-resistant acid phosphatase 5b, 25( $\mathrm{OH}) D$ 25-hydroxyl vitamin D, $P T H$ parathyroid hormone, YAM young adult mean, $F N$ femoral neck, $T P F$ total proximal femur

${ }^{*} P<0.05$ vs. zoledronic acid group; ${ }^{\dagger} P<0.05$ vs. BPs group
Fig. 3 Correlation between the changes in $\mathrm{Ca}$ from baseline to 1-2 weeks after administration, and total type 1 procollagen$\mathrm{N}$-propeptide and tartrate-resistant acid phosphatase 5b, P1NP: total type 1 procollagen- $N$-propeptide, TRACP-5b: tartrateresistant acid phosphatase $5 \mathrm{~b}$
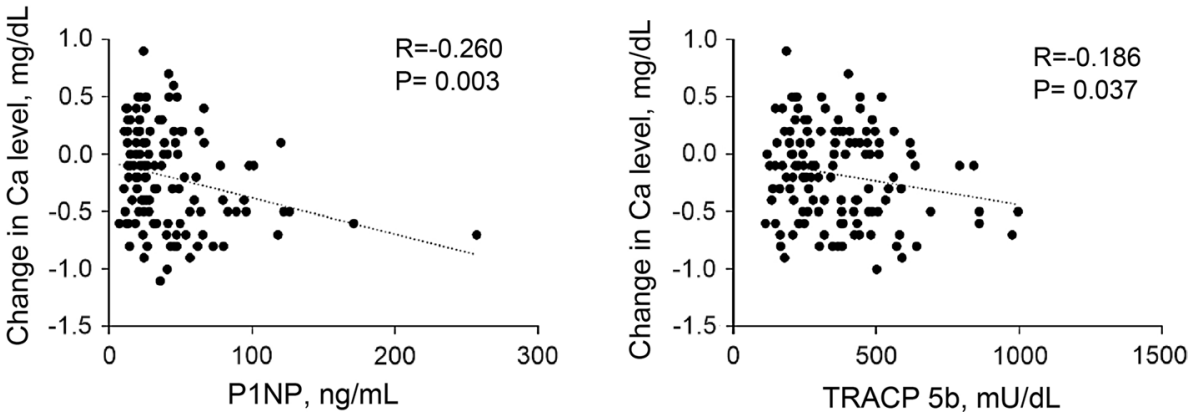

remained suppressed after 6 and 12 months (Fig. 4). Patients in the DMAb groups showed significant suppression of P1NP level at 12 months $(P<0.001)$ and TRACP-5b level at 6 and 12 months $(P=0.046$ and $P=0.003$, respectively) compared with those in the Zol groups. The NBP groups showed decreased P1NP and TRACP-5b levels at 6 and 12 months compared with the BP groups $(P<0.001)$.

\section{Longitudinal changes in BMD}

At 6 months after the administration of DMAb or Zol, all the groups showed approximately $2.5-4.3 \%$ increase in lumbar spine and femoral neck BMDs compared with the baseline (Fig. 5). All groups showed approximately $1.2-2.7 \%$ increase in total proximal femoral BMDs compared with the baseline. At 12 months, the DMAb groups exhibited a bigger increase in lumbar spine and total proximal femoral BMDs, albeit not significantly, compared with the Zol groups. There were no significant differences in the increase in BMDs at 12 months, between the BP and NBP groups. Total P1NP and TRACP-5b levels at baseline were associated with \%change in lumbar spine BMD from baseline to 12 months (total P1NP in Zol group: $\beta=0.339, P=0.024$ and DMAb group: $\beta=0.564$, $P<0.001)$ and TRACP-5b (Zol group: $\beta=0.311, P=0.036$ and DMAb group: $\beta=0.425, P=0.004$ ) (Fig. 6). However, bone metabolic markers were not associated with \%change in the femoral neck and total proximal femur BMDs from baseline to 12 months. 
Fig. 4 Comparison of changes in A serum P1NP levels and B serum TRACP-5b levels at 6 and 12 months post-administration. Data show mean \pm SEM, Zol: zoledronate acid, DMAb: denosumab, BPs: bisphosphonates, non-BPs: non-bisphosphonates, P1NP: total type 1 procollagen-N-propeptide, TRACP-5b: tartrate-resistant acid phosphatase $5 b$

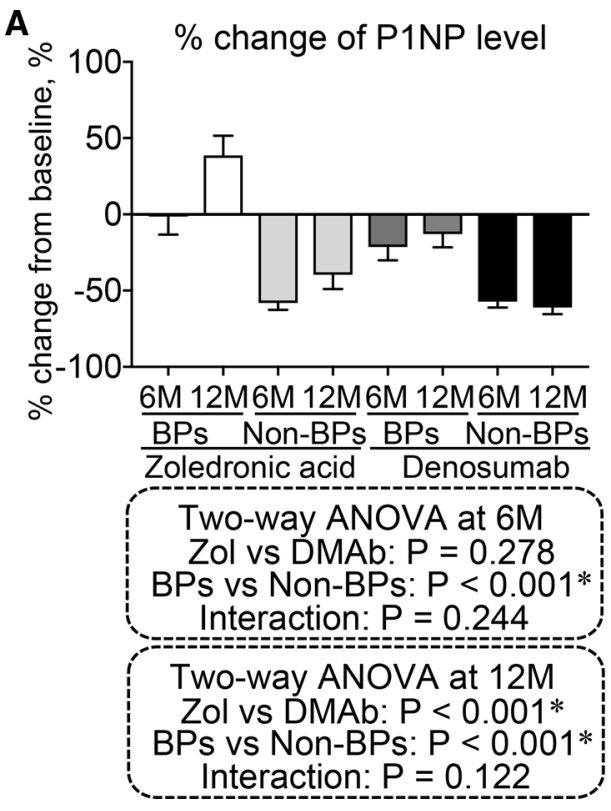

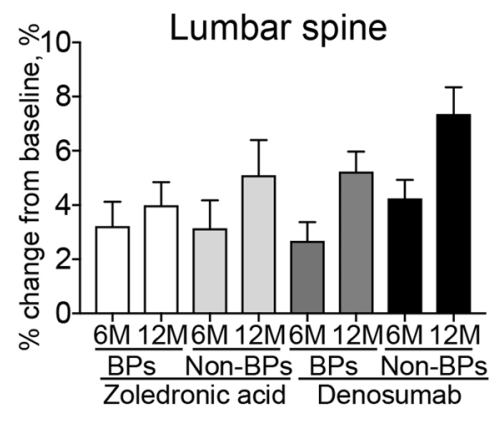
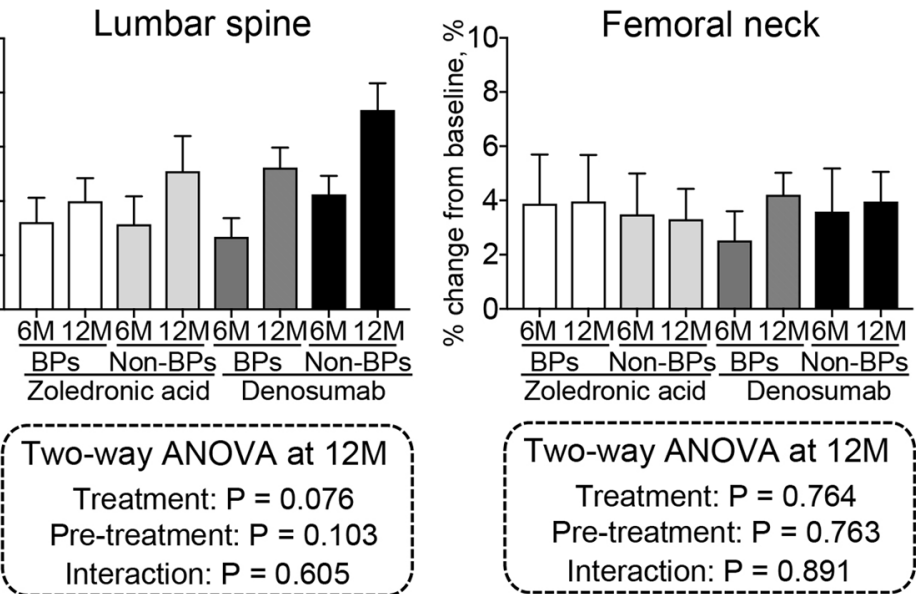

Zoledronic acid Denosumab

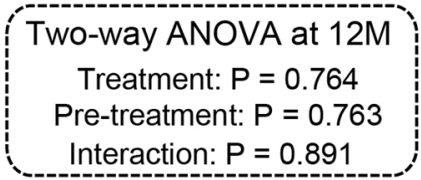

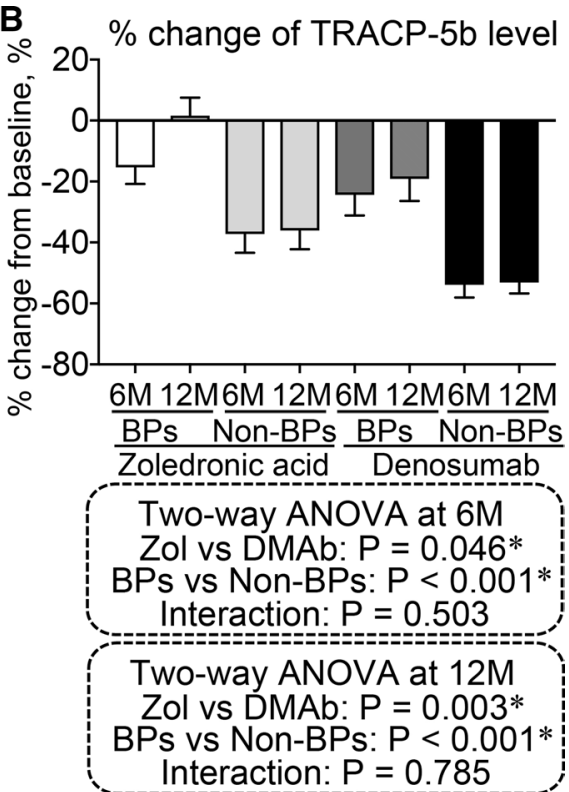

Fig. 5 Longitudinal changes in $\%$ change in bone mineral densities of lumbar, femoral neck, and total proximal femur. Data show mean \pm SEM

\section{Side effects and APRs}

During the observational period, there were no cases of necrosis of the jaw and atypical femoral fracture. Patients in the DMAb group did not have side effects, such as hypocalcemia or hypercalcemia, which required treatment. However, 17 of 75 patients treated using Zol experienced APRs. Although patients in the DMAb groups continued treatment for over 12 months, 8 of 73 patients in the Zol groups discontinued the treatment after 12 months due to side effects (including APRs) and drug eruption involving skin redness and wheal formation. Comparisons of the clinical characteristics at baseline are summarized in Table 2. Patients with APRs were younger than those without APRs $(P<0.001)$. Although the ratio of prior BP use was lower in patients with APRs compared to those without APRs, there were no significant differences in the ratio of prior use of active vitamin D and acetaminophen between patients with and without APRs. The mean P1NP level of patients with APRs at baseline was higher than that of patients without APRs $(P=0.002)$. The mean $25(\mathrm{OH}) \mathrm{D}$ level in patients with APRs at baseline was lower than that in patients without APRs $(P=0.019)$. Patients with APRs exhibited higher Ca depletion from baseline to 1-2 weeks compared to those without APRs $(P=0.030)$. Patients with APRs exhibited a larger increase in lumbar spine BMD from baseline to 12 months compared to those without APRs.

In the univariate analysis, age, prior BP use, P1NP level, and $25(\mathrm{OH}) \mathrm{D}$ level at baseline were identified as potential 
Fig. 6 Correlation between the changes in bone metabolic markers a total type 1 procollagen- $N$-propeptide and $\mathbf{b}$ tartrateresistant acid phosphatase $5 \mathrm{~b}$ and lumbar bone mineral density. Linear regression models adjusted for age, bone mineral density, pretreatment with bisphosphonate and active vitamin $\mathrm{D}, 25$-dihydroxyvitamin $\mathrm{D}$ levels, $\%$ young adult mean at baseline, and treatment

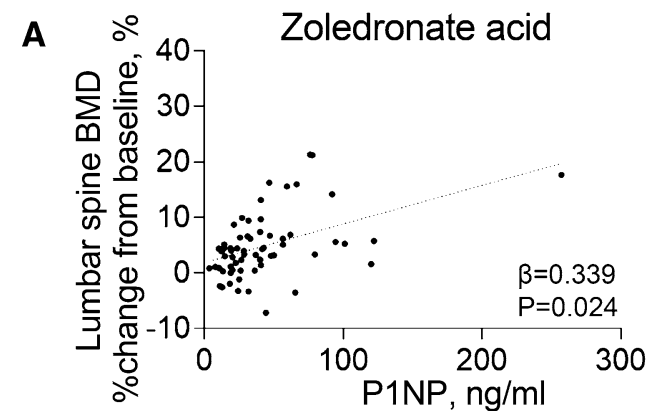

B

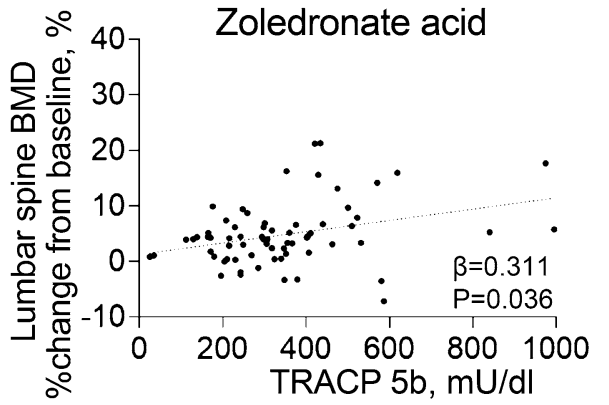

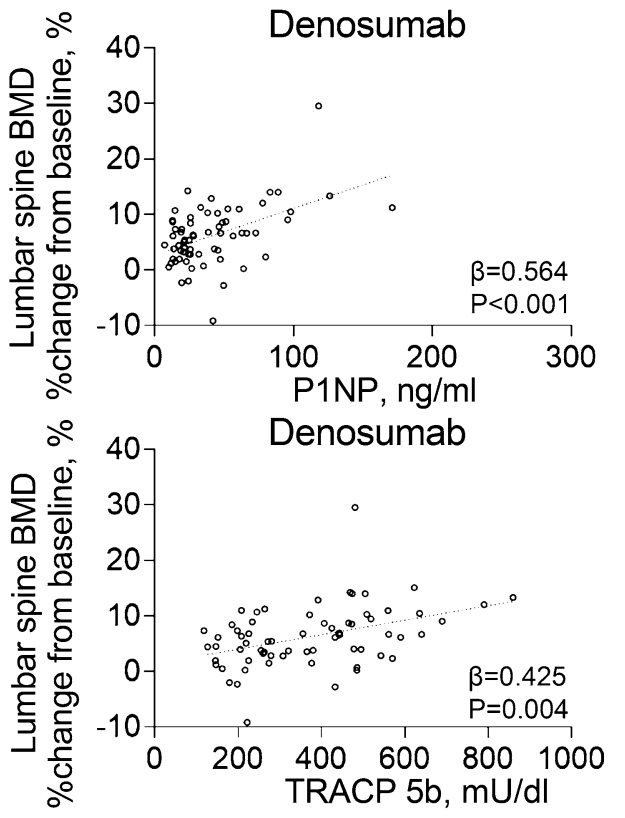

Table 2 Comparisons of clinical characteristics between patients with and without acute-phase reaction

\begin{tabular}{lllr}
\hline Variable & APRs $(+)$ & APRs $(-)$ & $P$ value \\
\hline Number & 17 & 58 & \\
Age (years) & $70.8(2.3)$ & $79.8(1.0)$ & $<0.001$ \\
BMI $\left(\mathrm{kg} / \mathrm{m}^{2}\right)$ & $21.6(0.8)$ & $22.7(0.5)$ & 0.273 \\
Prior active vitamin D3 use & 10 patients & 43 patients & 0.223 \\
Prior bisphosphonate use & 5 patients & 38 patients & 0.017 \\
Acetaminophen* use & 10 patients & 24 patients & 0.204 \\
Ca (mg/dL) & $9.37(0.13)$ & $9.35(0.05)$ & 0.885 \\
eGFR (mL/min/1.73 mm $\left.{ }^{2}\right)$ & $67.2(4.4)$ & $63.5(1.7)$ & 0.357 \\
P1NP (ng/mL) & $65.0(15.1)$ & $33.1(2.9)$ & 0.002 \\
TRACP-5b (mU/dL) & $390.0(51.6)$ & $337.1(22.8)$ & 0.324 \\
25(OH)D (ng/mL) & $10.7(1.2)$ & $14.8(0.9)$ & 0.019 \\
Intact PTH, (pg/mL) & $45.6(9.5)$ & $33.0(3.0)$ & 0.112 \\
Change in Ca levels (mg/dL) & $-0.45(0.09)$ & $-0.18(0.06)$ & 0.030 \\
$\%$ change in lumbar BMD & $6.96(1.95)$ & $3.57(0.71)$ & 0.045 \\
$\quad$ \%) & & & \\
\% change in FN BMD (\%) & $3.88(1.51)$ & $3.63(1.41)$ & 0.925 \\
\% change in TPF BMD TPF & $2.52(0.63)$ & $2.25(1.01)$ & 0.886 \\
$\quad$ (\%) & & & \\
\hline
\end{tabular}

Mean (standard error of the mean)

$A P R$ acute-phase reaction, $B M I$ body mass index, $C r$ creatinine, $e G F R$ estimated glomerular filtration rate, $P 1 N P$ total type 1 procollagen- $N$-propeptide, TRACP-5b tartrate-resistant acid phosphatase $5 \mathrm{~b}$, $25(\mathrm{OH}) \mathrm{D}$ 25-hydroxyl vitamin $\mathrm{D}, P T H$ parathyroid hormone, $B M D$ bone mineral density, $F N$ femoral neck, $T P F$ total proximal femur

*Patients took $200 \mathrm{mg}$ tablets three times a day after every meal
Table 3 Univariate and multivariate logistic regression analyses of factors affecting development of acute-phase reactions

\begin{tabular}{lllll}
\hline Factor & Odds ratio & $\begin{array}{l}95 \% \text { confi- } \\
\text { dence interval }\end{array}$ & $P$ value \\
\hline Univariate analysis & & & & \\
Age (years) & 0.875 & 0.810 & 0.945 & 0.001 \\
Prior active vitamin D3 use & 0.498 & 0.161 & 1.544 & 0.227 \\
Prior bisphosphonate use & 0.254 & 0.079 & 0.082 & 0.022 \\
Acetaminophen* use & 2.024 & 0.675 & 6.069 & 0.208 \\
P1NP (ng/mL) & 1.027 & 1.005 & 1.048 & 0.014 \\
TRACP-5b (mU/dL) & 1.001 & 0.999 & 1.004 & 0.325 \\
25(OH)D (ng/mL) & 0.874 & 0.776 & 0.984 & 0.026 \\
Intact PTH (pg/mL) & 0.984 & 0.960 & 1.005 & 0.148 \\
Multivariate analysis & & & & \\
P1NP (ng/mL) & 1.039 & 1.005 & 1.074 & 0.025 \\
TRACP-5b (mU/dL) & 1.001 & 0.997 & 1.005 & 0.640 \\
25(OH)D (ng/mL) & 0.851 & 0.736 & 0.983 & 0.029 \\
Intact PTH (pg/mL) & 0.998 & 0.967 & 1.026 & 0.911 \\
\hline
\end{tabular}

Mean (standard error of the mean)

$A P R$ acute-phase reaction, $P 1 N P$ total type 1 procollagen- $N$-propeptide, TRACP-5b tartrate-resistant acid phosphatase $5 \mathrm{~b}, 25(\mathrm{OH}) D$ 25-hydroxyl vitamin D, $P T H$ parathyroid hormone, $Y A M$ young adult mean, $F N$ femoral neck, $T P F$ total proximal femur

*Patients took $200 \mathrm{mg}$ tablets three times a day after every meal; \#Adjusted by age, prior active vitamin D3, bisphosphonate use, and acetaminophen use

predictors for the development of APRs (Table 3). Furthermore, the P1NP and 25(OH)D levels at baseline were identified as potential predictors of the development of APRs 
in the multivariate logistic regression analyses adjusted for age, prior use of active vitamin $\mathrm{D} 3, \mathrm{BP}$, and acetaminophen.

\section{Discussion}

This study showed that the combination therapy of Zol or DMAb and ELD increased lumbar spine and hip BMDs, regardless of pretreatment, thus suggesting that both combination therapies are effective treatments for Japanese patients with postmenopausal osteoporosis. Patients in the DMAb groups exhibited increased lumbar spine and total proximal femoral BMDs, albeit not significantly different compared to that in the Zol groups. This finding is slightly different from those of previous studies comparing DMAb and $\mathrm{Zol}[32,33]$. Since the effect of ELD on the bone is independent of its supplementary effect in vitamin D insufficiency [34], this discrepancy may be explained by the fact that all the patients received combination therapy with ELD.

In this study, patients in the BP-Zol group exhibited attenuation of suppression of bone turnover at 12 months, suppressed by pretreatment. This finding was consistent with those reported in previous studies $[33,35]$. McClung et al. [35] reported that transition to Zol from oral alendronate attenuated the suppression of bone turnover marker at 12 months; however, bone biopsies at 12 months exhibited decrease in excessive remodeling, as seen in osteoporosis. In this study, the BP-Zol group exhibited an increase in BMD at 12 months despite the attenuation in suppressed bone turnover. This finding supported the conclusion that patients can be switched from oral BPs to Zol infusion with maintenance of therapeutic effect for at least 12 months. However, because it is unknown whether this attenuation would have positive outcomes in preventing fragility fracture or severely suppressed bone turnover in the future, long-term continuous follow-up is necessary. Patients in the non-BPs groups exhibited increased lumbar spine, albeit not significant, compared with patients in the BPs groups. Further, bone metabolic markers at baseline were associated with changes in BMD from baseline to 12 months. Therefore, previous treatment regimen and bone metabolic markers at baseline may be useful for evaluating BMD during treatment with Zol or DMAb and ELD.

Although mean serum Ca levels decreased 1 or 2 weeks after the first administration of DMAb or Zol in combination with ELD, none of the patients experienced serious side effects related to hypocalcemia. There was a significant decrease in Ca levels in the NBP group compared to that in the BP group. Bone metabolic markers at baseline had significant correlations with changes in serum Ca levels from baseline to 1 or 2 weeks, similar to the results of a previous study regarding denosumab-induced hypocalcemia [36]. Although the DMAb group continued the treatment over 12 months, the Zol group experienced APRs ( $>20 \%$ ), and 8 of 73 patients discontinued Zol. Therefore, DMAb may have relatively fewer side effects and was easier to administer compared to Zol. However, a systematic review has reported increased risk of multiple vertebral fractures after discontinuation of DMAb [37]; and therefore, strict adherence may be required with DMAb.

Consistent with the previous reports [38, 39], we found that age, prior BP use, and lower 25(OH)D levels were associated with APRs. Considering the results from the multivariate analysis, higher P1NP level and lower 25(OH)D at baseline may be risk factors for APRs at the first administration and need to be monitored before the first administration. In contrast to previous reports [40, 41], this study showed that acetaminophen use could not prevent APRs. This discrepancy could be explained by the fact that the dose used in this study (200 mg tablets, three times a day) was lower than that used in the previous study [41]. A recent Japanese randomized study reported that Zol-induced APRs could be suppressed by non-steroidal anti-inflammatory drugs (NSAIDs) [28]. Therefore, to prevent these APRs, an appropriate dose of acetaminophen or NSAIDs after the administration may be important in addition to monitoring bone metabolic markers and 25(OH)D levels. Considering that the ratio of APRs $(17 / 75$ cases $=22.7 \%)$ in this study for all patients who received ELD after infusion of Zol was less, regardless of lower mean $25(\mathrm{OH}) \mathrm{D}$ level, compared with that in a previous report [42], ELD could also be effective in preventing APRs. Additionally, since patients with APRs exhibited a greater decrease in Ca levels from baseline to 1-2 weeks than those without APRs, patients with APRs should be strictly monitored for hypocalcemia. Moreover, considering that patients with APRs exhibited significant increase in BMD from baseline to 12 months compared to those without APRs, APRs might have reflected the reaction to the therapy.

There were some limitations in this study. First, this study had a small sample size and a short observation period. Further studies are needed to ascertain whether BMD continuously increases upon treatment with Zol or DMAb and ELD and to what extent fractures can be prevented. Second, this study included pretreatment with various BPs, including alendronate and risedronate, and pretreatment with active vitamin D3, such as alfacalcidol and ELD, which might have affected the results.

In conclusion, the combination therapy of Zol or DMAb and ELD may increase BMD at 12 months after the first administration, regardless of BP pretreatment, in Japanese patients with postmenopausal osteoporosis. Bone metabolic markers at baseline may be useful predictors for reaction to the therapy and side effects such as APRs and hypocalcemia during these combination therapies in postmenopausal 
osteoporosis. The prior use of BP is protective against the development of APR.

Acknowledgements This project was supported in part by a Grantin-Aid for Young Scientists from the Ministry of Education, Culture, Sports, Science, and Technology of Japan 20K17948 (T. Shimizu), and Japan Osteoporosis Foundation Grant for Bone Research (D. Takahashi).

\section{Declaration}

Conflict of interest Yumejiro Nakamura, Tomohiro Shimizu, Tsuyoshi Asano, Shun Shimodan, Hotaka Ishizu, Daisuke Takahashi, Masahiko Takahata and Norimasa Iwasaki declare that they have no conflict of interest.

\section{References}

1. Johnell O, Kanis JA (2006) An estimate of the worldwide prevalence and disability associated with osteoporotic fractures. Osteoporos Int 17:1726-1733. https://doi.org/10.1007/ s00198-006-0172-4

2. Who are candidates for prevention and treatment for osteoporosis? (1997). Osteoporos Int 7:1-6

3. Sambrook P, Cooper C (2006) Osteoporosis. Lancet 367:2010 2018. https://doi.org/10.1016/S0140-6736(06)68891-0

4. Silverman SL, Schousboe JT, Gold DT (2011) Oral bisphosphonate compliance and persistence: a matter of choice? Osteoporos Int 22:21-26. https://doi.org/10.1007/s00198-010-1274-6

5. Penning-van Beest FJ, Erkens JA, Olson M, Herings RM (2008) Loss of treatment benefit due to low compliance with bisphosphonate therapy. Osteoporos Int 19:511-517. https://doi.org/10.1007/ s00198-007-0466-1

6. Kanis JA, Cooper C, Hiligsmann M, Rabenda V, Reginster JY, Rizzoli R (2011) Partial adherence: a new perspective on health economic assessment in osteoporosis. Osteoporos Int 22:25652573. https://doi.org/10.1007/s00198-011-1668-0

7. Olsen KR, Hansen C, Abrahamsen B (2013) Association between refill compliance to oral bisphosphonate treatment, incident fractures, and health care costs-an analysis using national health databases. Osteoporos Int 24:2639-2647. https://doi.org/10.1007/ s00198-013-2365-y

8. Recker RR, Gallagher R, MacCosbe PE (2005) Effect of dosing frequency on bisphosphonate medication adherence in a large longitudinal cohort of women. Mayo Clin Proc 80:856-861. https:// doi.org/10.4065/80.7.856

9. Cotte FE, Fardellone P, Mercier F, Gaudin AF, Roux C (2010) Adherence to monthly and weekly oral bisphosphonates in women with osteoporosis. Osteoporos Int 21:145-155. https://doi.org/10. 1007/s00198-009-0930-1

10. Li Q, Guan X, Wu P, Wang X, Zhou L, Tong Y, Ren R, Leung KS, Lau EH, Wong JY, Xing X (2020) Early transmission dynamics in Wuhan, China, of novel coronavirus-infected pneumonia. N Engl J Med 382:1199-1207. https://doi.org/10.1056/NEJMoa2001316

11. Zhu N, Zhang D, Wang W, Li X, Yang B, Song J, Zhao X, Huang B, Shi W, Lu R, Niu P (2020) A novel coronavirus from patients with pneumonia in China, 2019. N Engl J Med 382:727-733. https://doi.org/10.1056/NEJMoa2001017

12. Lacey DL, Boyle WJ, Simonet WS, Kostenuik PJ, Dougall WC, Sullivan JK, San Martin J, Dansey R (2012) Bench to bedside: elucidation of the OPG-RANK-RANKL pathway and the development of denosumab. Nat Rev Drug Discov 11:401-419. https:// doi.org/10.1038/nrd3705
13. Brown JP, Prince RL, Deal C, Recker RR, Kiel DP, de Gregorio LH, Hadji P, Hofbauer LC, Alvaro-Gracia JM, Wang H, Austin M, Wagman RB, Newmark R, Libanati C, San Martin J, Bone HG (2009) Comparison of the effect of denosumab and alendronate on BMD and biochemical markers of bone turnover in postmenopausal women with low bone mass: a randomized, blinded, phase 3 trial. J Bone Miner Res 24:153-161. https://doi.org/10.1359/jbmr. 08090110.1359/jbmr.0809010

14. Brown JP, Roux C, Ho PR, Bolognese MA, Hall J, Bone HG, Bonnick S, van den Bergh JP, Ferreira I, Dakin P, Wagman RB, Recknor C (2014) Denosumab significantly increases bone mineral density and reduces bone turnover compared with monthly oral ibandronate and risedronate in postmenopausal women who remained at higher risk for fracture despite previous suboptimal treatment with an oral bisphosphonate. Osteoporos Int 25:19531961. https://doi.org/10.1007/s00198-014-2692-7

15. Roux C, Hofbauer LC, Ho PR, Wark JD, Zillikens MC, Fahrleitner-Pammer A, Hawkins F, Micaelo M, Minisola S, Papaioannou N, Stone M, Ferreira I, Siddhanti S, Wagman RB, Brown JP (2014) Denosumab compared with risedronate in postmenopausal women suboptimally adherent to alendronate therapy: efficacy and safety results from a randomized open-label study. Bone 58:4854. https://doi.org/10.1016/j.bone.2013.10.006

16. Maricic M (2010) The role of zoledronic acid in the management of osteoporosis. Clin Rheumatol 29:1079-1084. https://doi.org/ 10.1007/s10067-010-1486-3

17. Walker-Bone K (2011) Preventing fractures in the elderly. Br J Hosp Med (Lond) 72:576-581. https://doi.org/10.12968/hmed. 2011.72.10.576

18. Nakamura T, Fukunaga M, Nakano T, Kishimoto H, Ito M, Hagino H, Sone T, Taguchi A, Tanaka S, Ohashi M, Ota Y, Shiraki M (2017) Efficacy and safety of once-yearly zoledronic acid in Japanese patients with primary osteoporosis: two-year results from a randomized placebo-controlled double-blind study (ZOledroNate treatment in Efficacy to osteoporosis; ZONE study). Osteoporos Int 28:389-398. https://doi.org/10.1007/s00198-016-3736-y

19. Yoshizawa T, Nishino T, Okubo I, Yamazaki M (2018) Cost-effectiveness analysis of drugs for osteoporosis treatment in elderly Japanese women at high risk of fragility fractures: comparison of denosumab and weekly alendronate. Arch Osteoporos 13:94. https://doi.org/10.1007/s11657-018-0509-6

20. Asano T, Shimizu T, Takahashi D, Ota M, Sato D, Hamano H, Hiratsuka S, Takahata M, Iwasaki N (2019) Potential association with early changes in serum calcium level after starting or switching to denosumab combined with eldecalcitol. J Bone Miner Metab 37:351-357. https://doi.org/10.1007/s00774-018-0928-x

21. Ito M, Sone T, Shiraki M, Tanaka S, Irie C, Ota Y, Nakamura T (2018) The effect of once-yearly zoledronic acid on hip structural and biomechanical properties derived using computed tomography (CT) in Japanese women with osteoporosis. Bone 106:179186. https://doi.org/10.1016/j.bone.2017.10.013

22. Farinola N, Kanjanapan Y (2013) Denosumab-induced hypocalcaemia in high bone turnover states of malignancy and secondary hyperparathyroidism from renal failure. Intern Med J 43:12431246. https://doi.org/10.1111/imj.12283

23. Ungprasert $P$, Cheungpasitporn W, Srivali N, Kittanamongkolchai W, Bischof EF (2013) Life-threatening hypocalcemia associated with denosumab in a patient with moderate renal insufficiency. Am J Emerg Med 31:756 e1-2. https://doi.org/10.1016/j.ajem. 2012.11.011

24. Anastasilakis AD, Toulis KA, Polyzos SA, Anastasilakis CD, Makras P (2012) Long-term treatment of osteoporosis: safety and efficacy appraisal of denosumab. Ther Clin Risk Manag 8:295306. https://doi.org/10.2147/TCRM.S24239

25. Block GA, Bone HG, Fang L, Lee E, Padhi D (2012) A singledose study of denosumab in patients with various degrees of renal 
impairment. J Bone Miner Res 27:1471-1479. https://doi.org/10. 1002/jbmr.1613

26. Black DM, Delmas PD, Eastell R, Reid IR, Boonen S, Cauley JA, Cosman F, Lakatos P, Leung PC, Man Z, Mautalen C (2007) Once-yearly zoledronic acid for treatment of postmenopausal osteoporosis. N Engl J Med 356:1809-1822. https://doi.org/10. 1056/NEJMoa067312

27. Popp AW, Senn R, Curkovic I, Senn C, Buffat H, Popp PF, Lippuner K (2017) Factors associated with acute-phase response of bisphosphonate-naive or pretreated women with osteoporosis receiving an intravenous first dose of zoledronate or ibandronate. Osteoporos Int 28:1995-2002. https://doi.org/10.1007/ s00198-017-3992-5

28. Okimoto N, Sakai A, Yoshioka T, Kobayashi T, Asano K, Akahoshi S, Ishikura T, Fukuhara S, Fuse Y, Mizuno T, Katae Y (2020) Efficacy of non-steroidal anti-inflammatory drugs on zoledronic acid-induced acute-phase reactions: randomized, open-label, Japanese OZ study. J Bone Miner Metab 38:230-239. https://doi.org/ 10.1007/s00774-019-01050-8

29. Matsumoto T, Ito M, Hayashi Y, Hirota T, Tanigawara Y, Sone T, Fukunaga M, Shiraki M, Nakamura T (2011) A new active vitamin D3 analog, eldecalcitol, prevents the risk of osteoporotic fractures - a randomized, active comparator, double-blind study. Bone 49:605-612. https://doi.org/10.1016/j.bone.2011.07.011

30. Delmas PD, McClung MR, Zanchetta JR, Racewicz A, Roux C, Benhamou CL, Man Z, Eusebio RA, Beary JF, Burgio DE, Matzkin E, Boonen S (2008) Efficacy and safety of risedronate $150 \mathrm{mg}$ once a month in the treatment of postmenopausal osteoporosis. Bone 42:36-42. https://doi.org/10.1016/j.bone.2007.09.001

31. Ebina K, Hashimoto J, Shi K, Kashii M, Hirao M, Yoshikawa H (2015) Undercarboxylated osteocalcin may be an attractive marker of teriparatide treatment in RA patients: response to Mokuda. Osteoporos Int 26:1445. https://doi.org/10.1007/ s00198-014-2993-x

32. Sheedy KC, Camara MI, Camacho PM (2015) Comparison of the efficacy, adverse effects, and cost of zoledronic acid and denosumab in the treatment of osteoporosis. Endocr Pract 21:275-279. https://doi.org/10.4158/EP14106.OR

33. Miller PD, Pannacciulli N, Brown JP, Czerwinski E, Nedergaard BS, Bolognese MA, Malouf J, Bone HG, Reginster JY, Singer A, Wang C, Wagman RB, Cummings SR (2016) Denosumab or zoledronic acid in postmenopausal women with osteoporosis previously treated with oral bisphosphonates. J Clin Endocrinol Metab 101:3163-3170. https://doi.org/10.1210/jc.2016-1801

34. Matsumoto T, Kubodera N (2007) ED-71, a new active vitamin D3, increases bone mineral density regardless of serum $25(\mathrm{OH})$ D levels in osteoporotic subjects. J Steroid Biochem Mol Biol 103:584-586. https://doi.org/10.1016/j.jsbmb.2006.12.088
35. McClung M, Recker R, Miller P, Fiske D, Minkoff J, Kriegman A, Zhou W, Adera M, Davis J (2007) Intravenous zoledronic acid $5 \mathrm{mg}$ in the treatment of postmenopausal women with low bone density previously treated with alendronate. Bone 41:122-128. https://doi.org/10.1016/j.bone.2007.03.011

36. Ishikawa K, Nagai T, Sakamoto K, Ohara K, Eguro T, Ito H, Toyoshima Y, Kokaze A, Toyone T, Inagaki K (2016) High bone turnover elevates the risk of denosumab-induced hypocalcemia in women with postmenopausal osteoporosis. Ther Clin Risk Manag 12:1831-1840. https://doi.org/10.2147/TCRM.S123172

37. Tsourdi E, Langdahl B, Cohen-Solal M, Aubry-Rozier B, Eriksen EF, Guanabens N, Obermayer-Pietsch B, Ralston SH, Eastell R, Zillikens MC (2017) Discontinuation of Denosumab therapy for osteoporosis: a systematic review and position statement by ECTS. Bone 105:11-17. https://doi.org/10.1016/j.bone.2017.08. 003

38. Reid IR, Gamble GD, Mesenbrink P, Lakatos P, Black DM (2010) Characterization of and risk factors for the acute-phase response after zoledronic acid. J Clin Endocrinol Metab 95:4380-4387. https://doi.org/10.1210/jc.2010-0597

39. Bertoldo F, Pancheri S, Zenari S, Boldini S, Giovanazzi B, Zanatta M, Valenti MT, Dalle Carbonare L, Lo Cascio V (2010) Serum 25-hydroxyvitamin D levels modulate the acute-phase response associated with the first nitrogen-containing bisphosphonate infusion. J Bone Miner Res 25:447-454. https://doi.org/10.1359/jbmr. 090819

40. Silverman SL, Kriegman A, Goncalves J, Kianifard F, Carlson T, Leary E (2011) Effect of acetaminophen and fluvastatin on postdose symptoms following infusion of zoledronic acid. Osteoporos Int 22:2337-2345. https://doi.org/10.1007/s00198-010-1448-2

41. Wark JD, Bensen W, Recknor C, Ryabitseva O, Chiodo J 3rd, Mesenbrink P, de Villiers TJ (2012) Treatment with acetaminophen/paracetamol or ibuprofen alleviates post-dose symptoms related to intravenous infusion with zoledronic acid $5 \mathrm{mg}$. Osteoporos Int 23:503-512. https://doi.org/10.1007/s00198-011-1563-8

42. Catalano A, Morabito N, Atteritano M, Basile G, Cucinotta D, Lasco A (2012) Vitamin D reduces musculoskeletal pain after infusion of zoledronic acid for postmenopausal osteoporosis. Calcif Tissue Int 90:279-285. https://doi.org/10.1007/ s00223-012-9577-6

Publisher's Note Springer Nature remains neutral with regard to jurisdictional claims in published maps and institutional affiliations. 